\title{
Qualidade de vida em grupos de mulheres acometidas de câncer de mama
}

\section{Quality of life of groups of women who suffer from breast cancer}

\author{
Sandra Fiorelli de Almeida Penteado Simeão ${ }^{1}$ \\ Izabel Cristina Rossi Landro ${ }^{1}$ \\ Marta Helena Souza De Conti ${ }^{1}$ \\ Márcia Aparecida Nuevo Gatti ${ }^{1}$ \\ Willian Dávila Delgallo ${ }^{1}$ \\ Alberto De Vitta ${ }^{1}$
}

${ }^{1}$ Pró Reitoria de Pesquisa e Pós-Graduação,

Universidade Sagrado

Coração. Rua Irmã Arminda

10-50, Jardim Brasil.

17011-160 Bauru SP.

ssimeao@usc.br
Abstract This study sought to investigate the quality of life of women who suffer from breast cancer and are part of a support group, comparing the data gathered from mastectomized women who had breast reconstruction and women who had not had surgery. It also compared data of women who had quadrantectomy surgery but did not need reconstruction and those who had not had reconstruction surgery. It is a quantitative transversal study, and data was collected by application of a questionnaire to the respondents and by the SF36 of the WHO. Fifty women with average age of 57.2 years participated in the survey. The groups were compared against each other - two by two considering types of surgery, using the MannWhitney Test at a 5\% level of significance. The results showed that women who had quadrantectomy surgery and did not need reconstruction are those who had the highest average scores in all fields and therefore have the best quality of life. They are followed by the group of women who had a mastectomy and reconstruction. The mastectomized group of women who had not had reconstruction surgery were seen to have a very low level of quality of life. They are followed by the group of women who had quadrantectomy surgery and also had not had reconstruction.

Key words Breast cancer, Quality of life, Self-help groups
Resumo O objetivo deste estudo foi investigar a qualidade de vida de mulheres integrantes de um Grupo de Apoio acometidas de câncer de mama e comparar a qualidade apurada entre as mulheres mastectomizadas que fizeram reconstrução mamária com aquelas que não fizeram além daquelas submetidas à quadrantectomia que não necessitavam da reconstrução com aquelas que não fizeram a reconstrução. Trata-se de estudo transversal quantitativo, cuja coleta de dados se deu por meio da aplicação de questionário de caracterização das entrevistadas e pelo SF-36 da OMS. Participaram 50 mulheres com idade média de 57,2 anos. Os grupos foram comparados entre si - dois a doisconsiderando os tipos de cirurgia, por meio do Teste de Mann Whitney, ao nível de 5\% de significância. Os resultados apontaram que as mulheres que realizaram a cirurgia de quadrantectomia e não necessitaram de reconstrução são as que possuem os melhores escores médios em todos os domíniose, portanto, têm melhor qualidade de vida, seguido do grupo de mastectomizadas que fizeram reconstrução. O grupo das mastectomizadas que não fizeram a reconstrução tem um nível muito baixo de qualidade de vida, seguido pelo grupo das mulheres que realizaram a quadrantectomia e também não fizeram a reconstrução.

Palavras-chave Câncer de mama, Qualidade de vida, Grupos de Autoajuda 


\section{Introdução}

Segundo o Cancer Statistics, em 2008 houve 182.460 novos casos de câncer de mama em mulheres nos Estados Unidos e 40.480 mortes em decorrência dele ${ }^{1}$. O Instituto Nacional do Câncer, em estimativa feita para 2010, apontou que seriam diagnosticados no Brasil 49.240 casos novos, sendo a segunda neoplasia mais incidente em mulheres ${ }^{2}$. Com o aumento das taxas de detecção precoce e com a melhoria nos tratamentos oferecidos, sabe-se que, aproximadamente, $50 \%$ das mulheres sobreviverão por, pelo menos, 15 anos após o diagnóstico e deverão ajustar-se às sequelas cirúrgicas ${ }^{3}$.

O câncer de mama não é mais considerado uma doença fatal, mas crônica, por isso é importante saber como será a vida das mulheres após o tratamento ${ }^{4,5}$. Algumas pesquisas destacam o quão comuns são os problemas de ordem sexual, humor, relações familiares, imagem do próprio corpo, quadros emocionais como depressão, ansiedade, ideação suicida, insônia e medo, interferindo também nas atividades do cotidiano, podendo levar a mulher ao isolamento social ${ }^{6-10}$.

As consequências emocionais causadas pela retirada da mama são muito grandes e as mulheres que passam por esta situação necessitam de assistência adequada visando sua reintegração familiar e social, assim como sua adaptação à nova realidade. A partir do momento em que a mulher é submetida à mastectomia radical sua autoestima pode ser afetada, o que diminui o grau de satisfação com o seu corpo e influi negativamente na qualidade do relacionamento conjugal ${ }^{11}$. A reconstrução mamária devolve a feminilidade, melhora a autoimagem e o relacionamento sexual ${ }^{12-}$ ${ }^{14}$, permitindo que essas mulheres apresentem atitudes positivas e de satisfação com o corpo, recuperando sua qualidade de vida ${ }^{3,15,16}$.

A sobrevida livre de doença e a sobrevida global são os principais parâmetros empregados na avaliação dos resultados do tratamento oncológico. Entretanto, tornou-se evidente que eles são insuficientes ${ }^{17,18}$. Na comparação de diferentes opções terapêuticas, a Qualidade de Vida tornou-se um importante parâmetro, auxiliando médicos e pacientes a decidirem a terapia mais adequada sendo considerada como a terceira dimensão a ser avaliada, além da eficácia (modificação da doença pelo efeito da droga) e da segurança (reação adversa a drogas) ${ }^{19,20}$.

O termo Qualidade de Vida está relacionado ao impacto do estado de saúde sobre a capacidade do indivíduo de viver plenamente, entretanto estão incluídas nesta definição uma variedade potencial de condições que afetam sua percepção, seus sentimentos e comportamen-tos relacionados ao seu funcionamento diário, assim como à sua condição de saúde e intervenções médicas ${ }^{18}$.

O avanço na detecção e no tratamento do câncer, as atitudes sociais mais otimistas, o aumento do número de pessoas que sobreviveram à doença e a quantidade de anos, a preocupação com a autonomia, os direitos dos pacientes e os aspectos psicossociais, entre outros, permitiram destaque crescente na avaliação da qualidade de vida de pessoas tratadas de câncer ${ }^{21-23}$.

O significado do suporte social para a mulher com câncer de mama durante todo o processo da doença se reveste de importância, pois ela se vê sendo ajudada para não desistir. Dessa maneira, é necessário destacar o significado atribuído pelas mulheres com câncer de mama quando encontram outras que vivem ou vivenciaram a mesma experiência, ao participarem de grupos de apoio $^{24,25}$. Estes, encorajam a autoconfiança e a esperança e melhoram a moral, favorecendo o enfrentamento da doença e suas incertezas. A participação de doentes nesses grupos tem sido indicada para amenizar o sofrimento emocional que pode ocorrer após o diagnóstico ${ }^{26,27}$.

A experiência pessoal de participaçäo em Grupos de Apoio a mulheres portadoras de câncer de mama vem sendo de grande valia para a melhoria da qualidade de vida destas, no que concerne ao resgate de sua autoestima e da cidadania ${ }^{27}$.

Considerando tais aspectos, teve-se por objetivo investigar a qualidade de vida de mulheres acometidas de câncer de mama integrantes de um Grupo de Apoio, por meio do SF-36, e comparar a qualidade apurada entre as mulheres mastectomizadas que fizeram reconstrução mamária com aquelas que não a fizeram e as quadrantectomizadas que não necessitavam da reconstrução com as que não fizeram a reconstrução.

\section{Metodologia}

Tratou-se de um estudo transversal de natureza quantitativa, com coleta de dados por meio da aplicação de dois instrumentos: um questionário de caracterização das entrevistadas e o genérico da Organização Mundial de Saúde SF-36 ${ }^{28}$.

Para seleção das mulheres, optou-se pela amostragem por acessibilidade, sendo incluídas as que frequentavam as reuniões do Grupo de Apoio "Amigas do Peito" de Bauru. As entrevis- 
tas ocorreram no período de dezembro de 2009 a março de 2010 nas reuniões deste Grupo.

A amostra foi composta por 50 mulheres extraída de uma população de 90 com diagnóstico de câncer de mama, que passaram por intervenção cirúrgica e completaram o tratamento radioterápico e/ou quimioterápico há pelo menos um ano.

Os questionários foram respondidos após o aceite e a obtenção da autorização das participantes, por meio da assinatura do termo de consentimento livre e esclarecido (TCLE), onde ficava explícito que não haveria ônus algum e que a pesquisa seria realizada por meio de respostas aos dois questionários mencionados.

O questionário de caracterização permitiu a divisão das mulheres em quatro gupos: o primeiro constituído por 10 mulheres mastectomizadas que fizeram a reconstrução mamária (grupo 1); o segundo, por 11 mulheres mastectomizadas que não fizeram a reconstrução (grupo 2); o terceiro, por 14 mulheres que fizeram a quadrantectomia e não necessitavam da reconstrução (grupo 3), e o quarto, por 15 mulheres que fizeram a quadrantectomia e não fizeram a reconstrução (grupo 4).

O questionário SF-36 foi escolhido por apresentar questões genéricas, abrangendo tópicos de interesse para a pesquisa, além de sua simples interpretação, e por ser de fácil aplicação podendo atingir um número maior de mulheres. Este instrumento foi traduzido e validado no Brasil para avaliar a qualidade de vida em pacientes com artrite reumatóide e mostrou-se adequado às condições socioeconômicas e culturais da população brasileira ${ }^{29}$. O fato do questionário ser centrado no impacto da patologia sobre a qualidade de vida e não na patologia ou nos sinais clínicos é justamente o grande diferencial deste instrumento. Embora existam questionários específicos (por exemplo, o European Organization for Research and Treatment of Cancer-EORTC ${ }^{5}$ ), os mesmos não apresentam validação para a cultura brasileira.

O SF-36 consiste de duas partes, sendo a primeira - Dimensão A - para avaliar o Estado de Saúde (com questões relacionadas à mobilidade física, dor, sono, energia, isolamento social e reações emocionais) e a segunda parte - Dimensão B - para avaliar o impacto da doença na vida diária do paciente. Trata-se de um questionário multidimensional formado por 36 itens englobados em 8 domínios: Capacidade Funcional (10 itens) - avalia a presença e extensão de limitações relacionadas à capacidade física; Aspecto Físico (04 itens) - avalia as limitações quanto ao tipo e quantidade de trabalho, bem como o quanto essas limitações dificultam a realização do trabalho e das atividades da vida diária; Dor (02 itens) - avalia a presença de dor, sua intensidade e sua interferência nas atividades da vida diária; Estado Geral de Saúde (05 itens) - avalia como o paciente se sente em relação à sua saúde global; Vitalidade (04 itens) - considera o nível de energia e de fadiga; Aspecto Social (02 itens) - analisa a integração do indivíduo em atividades sociais; Aspecto Emocional (03 itens) - avalia o impacto de aspectos psicológicos no bem-estar do paciente; e Saúde Mental (05 itens) - inclui questões sobre ansiedade, depressão, alterações no comportamento ou descontrole emocional e bemestar psicológico.

As 36 questões são estruturadas em escalas, com várias possibilidades de pontuação, como por exemplo: 1 e 2 (Sim ou Não); 1, 2 e 3 (Sim dificulta muito, Sim dificulta um pouco, Não dificulta de modo algum); 1 a 5 (Excelente, Muito Boa, Boa, Ruim, Muito Ruim); e outras categorizações. A variação é de intensidade crescente ou decrescente, conforme a questão. Para a obtenção dos escores, as questões devem ser pontuadas de acordo com normas pré-estabelecidas. Posteriormente, os valores das questões são transformados em notas para cada um dos oito domínios. Assim, cada um desses componentes possui um escore, cuja pontuação varia de 0 a 100 , sendo zero o pior e 100 o melhor estado de qualidade de vida.

Cada domínio é analisado separadamente, não havendo um escore total. O SF-36 inclui, ainda, um item de avaliação das alterações de saúde ocorridas no período de um ano (Questão 2) e que, apesar de não ser pontuado, é utilizado para comparação da saúde em geral.

Os dados coletados foram submetidos à análise estatística descritiva e os grupos foram comparados entre si-dois a dois - considerando os tipos de cirurgia (mastectomia - grupos 1 e 2; quadrantectomia - grupos 3 e 4), por meio do Teste de Mann Whitney, ao nível de 5\% de significância.

Esta pesquisa seguiu as normas regulamentadoras em seres humanos, conforme a Resolução CNS 196/96 ${ }^{30}$, tendo sido aprovada pelo Comitê de Ética em Pesquisa da Universidade Sagrado Coração. 


\section{Resultados}

A distribuição das 50 participantes de acordo com o tempo de diagnóstico do câncer de mama evidenciou 6 mulheres (12\%) com o período de 1 a 2 anos; $16(32 \%)$ de 2 a 3 anos; $11(22 \%)$ de 3 a 6 anos; 6 (12\%) de 6 a 7 anos; 7 (14\%) de 7 a 9 anos e $4(8 \%)$ com mais de 10 anos de diagnóstico.

A média de idade das entrevistadas foi de 57,2 anos com desvio-padrão de 12,2 anos (idade mínima de 32 anos e máxima de 81).

O perfil das participantes, segundo estado civil, escolaridade e ocupação, coletado por meio do questionário de caracterização, está representado, por suas frequências absoluta e relativa, na Tabela 1. Foram encontradas maiores porcentagens entre as mulheres com relação conjugal estável (62\%), entre aquelas que apresentam ensino superior (52\%) e entre as que trabalham fora (40\%).

Sobre o tipo de cirurgia, foram encontradas 21 mulheres que fizeram a mastectomia: 10 com reconstrução (grupo 1) e 11 sem reconstrução (grupo 2); 29 passaram por quadrantectomia: 14 não necessitaram de reconstrução (grupo 3) e 15 necessitavam, mas não a fizeram (grupo 4).

Os resultados encontrados na aplicação do SF-36 estão apresentados nas Tabelas 2 (Dimensão A - saúde física), 3 (Dimensão B - aspectos psíquicos/sociais) e 4 (Questão 2).

Na Tabela 2 é possível observar que o grupo 3 apresentou os melhores escores médios para os quatro domínios da Dimensão A, enquanto o grupo 2 revelou ou piores valores. Na Tabela 3 os piores escores médios também são do grupo

Tabela 1. Distribuição das 50 mulheres tratadas de câncer de mama segundo estado civil, escolaridade e profissão.

\begin{tabular}{lll}
\hline \multicolumn{1}{c}{ Estado civil } & \multicolumn{1}{c}{ Escolaridade } & \multicolumn{1}{c}{ Ocupação } \\
\hline Relação Conjugal Estável $=34(68 \%)$ & Ensino Fundamental $=11(22 \%)$ & Trabalha fora $=20(40 \%)$ \\
Solteira $=2(4 \%)$ & Ensino Médio $=13(26 \%)$ & Aposentada $=17(34 \%)$ \\
Divorciada $=9(18 \%)$ & Ensino Superior $=26(52 \%)$ & Do Lar $=13(26 \%)$ \\
Viúva $=5(10 \%)$ & & \\
\hline
\end{tabular}

Tabela 2. Medidas descritivas da Dimensão A apuradas no SF-36 das 50 entrevistadas em relação aos diferentes domínios e resultado do teste estatístico.

\begin{tabular}{|c|c|c|c|c|c|c|}
\hline Domínios & Grupos* & Média & Desvio-padrão & Mínimo & Máximo & Teste de Mann Whitney \\
\hline Capacidade & $1(\mathrm{n}=10)$ & 85,0 & 13,5 & 60 & 100 & \multirow{2}{*}{$p<0,05^{* *}$} \\
\hline \multirow[t]{3}{*}{ Funcional } & $2(\mathrm{n}=11)$ & 51,8 & 21,0 & 15 & 90 & \\
\hline & $3(\mathrm{n}=14)$ & 85,7 & 19,7 & 20 & 100 & \multirow{2}{*}{$p>0,05$} \\
\hline & $4(\mathrm{n}=15)$ & 78,0 & 20,1 & 25 & 100 & \\
\hline Limitação por & $1(\mathrm{n}=10)$ & 55,0 & 43,8 & 0 & 100 & \multirow{2}{*}{$p>0,05$} \\
\hline \multirow[t]{3}{*}{ Aspectos Físicos } & $2(\mathrm{n}=11)$ & 34,1 & 40,7 & 0 & 100 & \\
\hline & $3(\mathrm{n}=14)$ & 87,5 & 32,2 & 0 & 100 & \multirow{2}{*}{$p>0,05$} \\
\hline & $4(\mathrm{n}=15)$ & 75,7 & 38,8 & 0 & 100 & \\
\hline \multirow[t]{4}{*}{ Dor } & $1(\mathrm{n}=10)$ & 72,8 & 22,6 & 41 & 100 & \multirow{4}{*}{$p<0,05^{* *}$} \\
\hline & $2(\mathrm{n}=11)$ & 48,7 & 14,9 & 31 & 74 & \\
\hline & $3(\mathrm{n}=14)$ & 75,6 & 19,7 & 41 & 100 & \\
\hline & $4(\mathrm{n}=15)$ & 59,0 & 18,8 & 41 & 100 & \\
\hline \multirow{4}{*}{$\begin{array}{l}\text { Estado geral } \\
\text { de saúde }\end{array}$} & $1(\mathrm{n}=10)$ & 74,9 & 16,6 & 37 & 95 & \multirow{2}{*}{$p>0,05$} \\
\hline & $2(\mathrm{n}=11)$ & 63,0 & 19,4 & 35 & 92 & \\
\hline & $3(\mathrm{n}=14)$ & 77,7 & 13,1 & 42 & 97 & \multirow{2}{*}{$p>0,05$} \\
\hline & $4(\mathrm{n}=15)$ & 64,6 & 23,1 & 0 & 87 & \\
\hline
\end{tabular}

* Grupo 1: mastectomizadas com reconstrução; Grupo 2: mastectomizadas sem reconstrução; Grupo 3: quadrantectomizadas que não necessitaram de reconstrução; Grupo 4: quadrantectomizadas que necessitavam, mas não fizeram a reconstrução. ${ }^{* *}$ Diferença estatisticamente significante. 
2, enquanto os melhores estão alternados entre os outros 3 grupos.

A comparação entre os grupos apontou que os escores médios do grupo 1 sempre foram superiores aos do grupo 2, aparecendo diferença estatisticamente significante nos domínios $\mathrm{Ca}$ pacidade Funcional e Dor, observados na Tabela 2, e Vitalidade e Limitação por Aspectos Sociais Tabela 3.

$\mathrm{Na}$ análise comparativa entre os grupos 3 e 4, percebeu-se que na Dimensão A (Tabela 2), embora os escores médios do grupo 3 tenham sido superiores aos do grupo 4, a diferença estatisticamente significante foi percebida apenas no domínio Dor. Na Tabela 3, o escore médio do domínio Aspecto Emocional do grupo 3 foi inferior ao do grupo 4, sendo superior nos demais, com diferença estatisticamente significante no domínio Saúde Mental. Quando se traça um comparativo entre os domínios da Dimensão A (Tabela 3 ), percebe-se que os escores médios mais baixos encontram-se nos Aspectos Físicos para os grupos 1 e 2 e Dor para os grupos 3 e 4 . A mesma avaliação para a Dimensão B (Tabela 4), apontou que os piores escores médios estão relacionados aos domínios Vitalidade e Aspecto Emocional para os grupos 1 e 2 e Vitalidade para os grupos 3 e 4 . Tanto para uma Dimensão quanto para outra, não há predominância de qualquer domínio para os escores mais altos.

Em relação aos resultados da Questão 2 (Tabela 4) pode-se verificar que $60 \%$ das mulheres

Tabela 3. Medidas descritivas da Dimensão B apuradas no SF-36 das 50 entrevistadas em relação aos diferentes domínios.

\begin{tabular}{lcccccc}
\hline \multicolumn{1}{c}{ Domínios } & Grupos $^{*}$ & Média & Desvio-padrão & Mínimo & Máximo & Teste de Mann Whitney \\
\hline Vitalidade & $1(\mathrm{n}=10)$ & 68,5 & 6,7 & 55 & 75 & $p<0,05^{* *}$ \\
& $2(\mathrm{n}=11)$ & 58,4 & 10,2 & 40 & 70 & \\
& $3(\mathrm{n}=14)$ & 66,1 & 11,1 & 30 & 75 & $p>0,05$ \\
& $4(\mathrm{n}=15)$ & 63,1 & 9,6 & 36 & 75 & \\
Limitação por & $1(\mathrm{n}=10)$ & 87,5 & 18,6 & 50 & 100 & $p<0,05^{* *}$ \\
Aspectos Sociais & $2(\mathrm{n}=11)$ & 65,9 & 20,1 & 37,5 & 100 & \\
& $3(\mathrm{n}=14)$ & 90,2 & 20,3 & 25 & 100 & \\
& $4(\mathrm{n}=15)$ & 79,2 & 20,8 & 38 & 100 & \\
Aspecto & $1(\mathrm{n}=10)$ & 70,0 & 42,9 & & & \\
Emocional & $2(\mathrm{n}=11)$ & 54,5 & 47,8 & 0 & 100 & \\
& $3(\mathrm{n}=14)$ & 80,9 & 28,5 & 33 & 100 & \\
& $4(\mathrm{n}=15)$ & 84,4 & 30,5 & 0 & 100 & \\
Saúde Mental & $1(\mathrm{n}=10)$ & 78,0 & 10,7 & 56 & 92 & \\
& $2(\mathrm{n}=11)$ & 71,6 & 15,5 & 36 & 88 & $p>0,05$ \\
& $3(\mathrm{n}=14)$ & 86,0 & 21,2 & 16 & 100 & \\
& $4(\mathrm{n}=15)$ & 78,4 & 15,3 & 52 & 100 & $p<0,05^{* *}$ \\
\hline
\end{tabular}

* Grupo 1: mastectomizadas com reconstrução; Grupo 2: mastectomizadas sem reconstrução; Grupo 3: quadrantectomizadas que não necessitaram de reconstrução; Grupo 4: quadrantectomizadas que necessitavam, mas não fizeram a reconstrução. ${ }^{* *}$ Diferença estatisticamente significante.

Tabela 4. Resultados da Questão 2 quanto a avaliação do indivíduo de sua saúde em relação há um ano.

\begin{tabular}{lcccc}
\hline Grupos $^{*}$ & $\mathbf{1}$ - Muito melhor & 2-Um pouco melhor $\mathbf{3}-$ Quase a mesma & 4 - Um pouco pior & Teste de Mann Whitney \\
\hline Grupo 1 & $6(60,0 \%)$ & $2(20,0 \%)$ & $1(10,0 \%)$ & $1(10,0 \%)$ \\
Grupo 2 & - & $4(36,4 \%)$ & $2(18,2 \%)$ & $5(45,5 \%)$ \\
Grupo 3 & $7(50,0 \%)$ & $1(7,1 \%)$ & $4(28,6 \%)$ & $2(14,3 \%)$ \\
Grupo 4 & $2(13,3 \%)$ & $3(20,0 \%)$ & $6(40,0 \%)$ & $4(26,7 \%)$
\end{tabular}

* Grupo 1: mastectomizadas com reconstrução; Grupo 2: mastectomizadas sem reconstrução; Grupo 3: quadrantectomizadas que não necessitaram de reconstrução; Grupo 4: quadrantectomizadas que necessitavam, mas não fizeram a reconstrução. ${ }^{* *}$ Diferença estatisticamente significante. 
do grupo 1 e 50\% do grupo 3, estavam "Muito melhores" que há um ano, o mesmo não acontecendo para os grupos 2 e 4 onde prevaleceram as respostas "Um pouco pior" (45,5\%) e "Quase a mesma" (40\%), respectivamente. Percebe-se também que há diferença estatisticamente significante entre os grupos 1 e 2 .

\section{Discussão}

Pelas avaliações dos grupos observou-se claramente que mulheres mastectomizadas que fizeram a reconstrução mamária (grupo 1) apresentaram melhores escores médios em todos os aspectos avaliados, quando comparadas com as mulheres que não realizaram a reconstrução (grupo 2). O grupo 3 também foi superior ao grupo 4 em todos os domínios, exceto no Aspecto Emocional.

Embora a análise dos domínios não permita associar diretamente os melhores escores de qualidade de vida com a percepção da imagem corporal, pode-se inferir que, quanto menos mutiladoras forem as consequências da cirurgia, mais qualidade de vida a mulher desfrutará, o que foi observado também por outros autores ${ }^{5,8,10-13}$. Além de afetar o psicológico de suas vítimas, fragilizando-as, a retirada parcial ou total de um órgão, como a mama, pode trazer agravos à satisfação sexual, à prática de esportes, à realização de atividades domésticas e ao convívio familiar, principalmente ao se considerar as funções sociais da mama, seu universo simbólico e sua identidade, que é essencialmente feminina, como a amamentação ${ }^{16,23,27,31}$. Assim, uma alteração na estrutura física, como a retirada da mama, representa não só uma alteração funcional, mas também sociocultural e psicológica para o indivíduo e o ambiente, repleta de simbolismos e significados que interferem diretamente na autoestima da mulher ${ }^{15,32,33}$.

Analisando-se a Tabela 2, verificou-se que nos domínios Capacidade Funcional e Dor existe diferença estatisticamente significante $(p<0,05)$ entre os grupos 1 e 2. Também foi observada diferença significante no aspecto Dor, para os grupos 3 e 4 .

Em estudo de corte transversal com amostra de 75 mulheres brasileiras avaliadas por meio do SF-36, os autores mostraram que houve um declínio nos Aspectos Físicos da qualidade de vida por referirem frequentemente dor, alterando a percepção de bem estar destas ${ }^{17}$. Em outro estudo, que também utilizou o SF-36, verificou-se que as mulheres relataram impacto negativo na qualidade de vida nos domínios Aspectos Físicos, Dor e Vitalidade ${ }^{21}$, assim como as mulheres mastectomizadas apresentaram piores escores de qualidade de vida, com significância estatística nos domínios Capacidade Funcional e Dor, em relação às submetidas à quadrantectomia ${ }^{32}$.

Pesquisa realizada utilizando o questionário EORTC, com 990 mulheres pós-tratamento de câncer de mama submetidas à mastectomia, também mostrou piora na Capacidade Funcional com limitações no trabalho e nos hábitos diários ${ }^{5}$.

Contrariando os resultados mencionados anteriormente, a qualidade de vida de 76 mulheres mastectomizadas que realizaram a reconstrução foi comparada com a de 920 mulheres suecas extraídas de uma população sem diagnóstico de câncer de mama, por meio do SF-36. Os resultados apontaram que os escores médios da Capacidade Funcional dos dois grupos foram similares e próximos de 90, o mesmo não acontecendo com a Dor. As mulheres do estudo tiveram menos dor do que as da população sadia, levando a crer que aquelas que se submetem à reconstrução podem ser fisicamente mais ativas do que outras mulheres e podem representar um subconjunto mais saudável do que a média da população ${ }^{16}$.

Embora não tenha sido observada diferença estatisticamente significante no domínio Limitação por Aspectos Físicos (Tabela 2), este apresentou os escores médios mais baixos entre todos os 8, para os grupos 1 e 2, sendo uma evidência do impacto negativo que a mastectomia traz fisicamente à mulher, mesmo para aquelas que realizaram a reconstrução. Alguns trabalhos enfatizam estes resultados, tendo sido encontrado baixo escore para este domínio mesmo após um ano da realização da cirurgia, assim como o encontro de aspectos negativos em momentos posteriores ao término do tratamento ${ }^{16,34}$. Foram referidos comprometimentos no domínio de Limitação por Aspectos Físicos, com o menor escore de todos os domínios avaliados, devido aos problemas como linfedema, dor, parestesias, diminuição da força muscular e redução da amplitude de movimento articular (ADM) do membro envolvido, que são frequentemente observados e relatados pelas mulheres operadas da mama, e merecem atenção, já que interferem na qualidade de vida delas ${ }^{21,35}$. Um estudo que avaliou a qualidade de vida de 73 mulheres, por meio do WHOQOL-Bref, chegou à mesma conclusão quanto ao domínio das limitações por aspectos físicos, assim como outro encontrou os piores 
escores de qualidade de vida associados à presença de mastectomia ${ }^{9,20}$.

Ainda em relação aos Aspectos Físicos, os escores médios dos grupos 3 e 4 superaram em muitos pontos percentuais os valores obtidos nos grupos 1 e 2, ou seja, pode-se inferir que os problemas físicos provocados pela mastectomia são realmente irreparáveis. A fraqueza muscular é uma das complicações da cirurgia para o tratamento de câncer de mama que é justificada pela retirada do músculo peitoral maior e/ou menor, pela fadiga e pela presença de linfedema. Este, além de ter um efeito estético, pode comprometer a execução de tarefas cotidianas que exijam força física e movimentos repetitivos, fato que pode gerar grande sofrimento à mulher que o vivencia ao se perceber impossibilitada de desempenhar algumas atividades que lhe traziam status social ou sustento financeiro. Portanto, a imagem corporal envolve aspectos fisiológicos, psicológicos e sociais que afetam as emoções, os pensamentos e o modo das pessoas se relacionarem com as outras ${ }^{15,36}$.

$\mathrm{Na}$ análise do domínio Estado Geral de Saúde (Tabela 2), não foi encontrada diferença significante para quaisquer grupos, os escores médios estão todos acima de 63 e, ainda, $76 \%$ das mulheres afirmaram que, comparada há um ano atrás, sua saúde está muito melhor, um pouco melhor ou quase a mesma (Tabela 4). Tais resultados estão em concordância com um trabalho, no qual foram encontrados escores próximos deste valor no momento pré-operatório, após um ano e para o grupo controle ${ }^{16}$. Em outras pesquisas foi mencionado o escore médio de 64,15 , assim como as participantes demonstraram melhores escores de qualidade de vida, principalmente quando associadas à cirurgia com conservação mamária ${ }^{10,17,21}$.

$\mathrm{Na}$ Tabela 3, onde se encontra o domínio Vitalidade, verificou-se diferença estatisticamente significante $(\mathrm{p}<0,05)$ entre os grupos 1 e 2 , sendo que o grupo 1 apresenta escore superior aos dos outros 3. Tal aspecto está diretamente relacionado ao vigor, à energia, à disposição e à força, ou seja, uma correspondência direta com os aspetos físicos, que, como discutido anteriormente, são impactantes para mulheres que passaram por uma mastectomia ou quadrantectomia, razão pela qual a literatura associa tais aspectos, como, por exemplo, a relação entre o desempenho sexual positivo das mulheres pesquisadas à vitalidade ${ }^{7}$. Contrariando tais resultados, alguns autores afirmam que há uma forte relação negativa entre o aspecto Vitalidade e o tratamento de esvaziamento axilar ${ }^{17,35,36}$, bem como ficou de- monstrado que mulheres submetidas à mastectomia com reconstrução imediata da mama apresentaram maiores distúrbios de bem-estar, humor e vitalidade ${ }^{37}$.

Ainda na Tabela 3, no domínio Limitação por Aspectos Sociais, verificou-se diferença estatisticamente significante $(\mathrm{p}<0,05)$ entre os grupos 1 e 2, tendo o grupo 1 apresentado melhor escore. Observa-se que neste domínio há forte influência dos resultados estéticos da cirurgia que proporcionam problemas com a imagem corporal, assim como preocupações com os aspectos financeiros e com a saúde futura podendo provocar maior estresse emocional, comprometendo a vida social. Entretanto, também é importante enfatizar que estas situações de desconforto estético podem ser minimizadas se as mulheres tiverem apoio social e familiar, assim como dos profissionais de saúde. Tais resultados podem ser comparados à pesquisa que observou 152 pacientes pareadas pelos tipos de cirurgia: conservadora ou radical da mama, onde o grupo de pacientes submetidas à mastectomia se mostrou menos satisfeito com o resultado estético da cirurgia e apresentou dificuldades emocionais ${ }^{38}$. Outros dois estudos, confirmam que mulheres mastectomizadas, quando comparadas com outros grupos submetidos a diferentes cirurgias, apresentaram problemas com a imagem corporal e evitavam ir à praia, manifestando uma tendência ao isolamento social ${ }^{15,39}$.

Em um trabalho com 110 participantes selecionadas no ambulatório de Oncologia Mamária do Centro de Atenção Integral à Saúde da Mulher (CAISM) da Universidade Estadual de Campinas (UNICAMP), notou-se que em relação aos escores de qualidade de vida, não houve diferença significativa nos domínios físico e de relações sociais quando analisados por faixa etária, nível de escolaridade, tipo de cirurgia e tempo decorrido desde a cirurgia. A importância do apoio à mulher com câncer de mama pode ser observada neste estudo, pois mulheres com companheiro apresentaram escores significativamente melhores nos domínios psicológico e relações sociais ${ }^{10}$. Também foi verificado que aquelas cuja avaliação da qualidade de vida foi positiva, tiveram apoio social, familiar e de profissionais ${ }^{23}$.

A análise do domínio Aspectos Emocionais permite observar que houve homogeneidade nos escores médios, com valores maiores que 70, para os grupos 1, 3 e 4. O grupo 2 apresentou o nível mais baixo, permitindo inferir que, do ponto de vista emocional, quanto menos mutilante for o resultado da cirurgia, melhor será o desempenho 
neste domínio. Tais resultados estão em concordância com as pesquisas que compararam grupos de mastectomizadas com reconstrução e sem reconstrução, sendo observada maior satisfação do grupo que realizou a reconstrução, sendo enfatizado que as mulheres que não realizaram a reconstrução tendem a apresentar o desejo de fazer a cirurgia estética, visando o reconhecimento do próprio corpo como novamente inteiro, com posterior consequências para os aspectos emocionais $^{13,33}$. Algumas mulheres mastectomizadas com ou sem reconstrução, que necessitaram de tratamento complementar, relataram bom nível emocional, apesar da diminuição da função física, e salientaram que intervenções clínicas para tratar sintomas comuns associados aos tratamentos, foram considerados diferenciais para a melhoria dos aspectos físico e emocional ${ }^{9}$.

Contrariando os resultados apontados acima, mulheres que fizeram a reconstrução mamária afirmaram não terem reconhecido o seio reconstruído como parte integrante de seu corpo e levaram um tempo para se adaptar à nova realidade, mesmo quando a reconstrução foi imediata, prejudicando as respostas a este domínio ${ }^{14,16}$.

Com relação ao domínio da Saúde Mental, observaram-se escores superiores a 71 e, ainda, diferença significativa ente os grupos 3 e 4 mostrando que mulheres do grupo 3 se encontravam em melhores condições. Entende-se por saúde mental a sensação de estar bem consigo mesmo e com os outros e a capacidade de lidar de forma positiva com as adversidades, sofrendo influências dos aspectos social e físico, espiritualidade e comunicação com os prestadores de serviços médicos. Resultados semelhantes a estes foram encontrados em alguns trabalhos nos quais mulheres que realizaram a cirurgia conservadora não apresentaram impacto negativo na Saúde Men$\mathrm{tal}^{38}$. Por meio da utilização de uma escala de satisfação com a vida e análise de seus domínios identificou-se que a Saúde Mental representa $70,8 \%$ da variação positiva entre os grupos de mulheres com câncer de mama estudados ${ }^{6}$.

Como já observado, a Tabela 3 relaciona os Aspectos Psíquicos/Sociais da qualidade de vida. É importante salientar que, em média, as respostas a estes aspectos tiveram escores superiores aos aspectos físicos. Pode-se atribuir tal condição ao fato das mulheres entrevistadas pertencerem ao Grupo de Apoio “Amigas do Peito". Os grupos funcionam como sustentáculo para dar continuidade ao processo de recuperação e adaptação à nova condição, e ainda como ambiente de transformação psicofísico e psicossocial. Tais
Grupos são um meio de instrumentalizar a independência, a autoestima, a identidade do ser cidadã e a qualidade de vida daquelas que os compõe, assim como constituírem-se fontes de suporte social às mulheres com câncer de mama ${ }^{24,25}$.

Um estudo realizado na Universidade Federal do Ceará aponta os efeitos benéficos na reabilitação de mulheres mastectomizadas que participam do GEPAM (Grupo de Auto-ajuda, Ensino, Pesquisa e Assistência à Mulher Mastectomizada - Universidade Federal do Ceará), reforçando o auxílio fornecido por este nas situações de estresse e medo, complementando o suporte da família, dos amigos e da religião como sistema de apoio no desempenho dos novos papéis assumidos posteriormente ao diagnóstico do câncer de mama $^{26}$.

Após o tratamento do câncer, algumas das iniciativas das mulheres relacionam-se à dedicação a obras sociais, maior envolvimento religioso e atuação como voluntárias em grupos de apoio a mulheres mastectomizadas. Tais ações são elementos considerados essenciais para o crescimento e/ou transformação do comportamento, levando à melhora da autoestima e da qualidade de vida ${ }^{27}$.

A Tabela 4 apresenta a Questão 2 do Questionário SF-36 que não faz parte do cálculo de qualquer domínio, sendo utilizada somente para avaliar o quanto o indivíduo está melhor ou pior comparado há um ano. Pode-se observar melhor porcentagem nos grupos 1 e 3, sendo compatível ao estudo que apresentou melhor adaptação à nova realidade entre os grupos de mastectomizadas que fizeram reconstrução e de mulheres que fizeram a cirurgia conservadora ${ }^{33}$.

\section{Considerações finais}

Os resultados do presente estudo mostraram que os melhores escores médios quanto aos domínios da Dimensão A foram do grupo 3, ou seja, mulheres que fizeram a quadrantectomia e não necessitavam da reconstrução, o mesmo não se repetindo na Dimensão B. Os menores escores médios para todos os oito domínios foram identificados para o grupo 2, mulheres mastectomizadas que não fizeram a reconstrução. Os grupos 1 e 3 tiveram melhores escores em todos os domínios, na comparação com os grupos 2 e 4 .

A comparação entre os grupos de mulheres mastectomizadas ( 1 e 2), apontou que o grupo 1 teve todos os escores superiores ao grupo 2, demonstrando uma melhor qualidade de vida das 
mulheres que se submeteram à reconstrução da mama. Para os grupos 3 e 4, os resultados dos escores demonstraram superioridade do grupo 3 em todos os valores, à exceção dos Aspectos Emocionais, onde o grupo 4 teve uma leve elevação.

Assim, conclui-se que a manutenção de um estado estético mais próximo da normalidade é determinante para a qualidade de vida destas mulheres. A análise dos domínios possibilitou compreender as dificuldades, nas dimensões analisadas, que o tipo de cirurgia impõe a estas mulheres.

Considera-se uma limitação desse estudo o número reduzido de mulheres, contudo, os resultados corroboraram com os da literatura, demonstrando a importância de estudos dessa natureza.

Um ponto favorável desse estudo foi que as mulheres questionadas eram todas integrantes da Associação “Amigas do Peito” de Bauru (SP), possuindo características bastante semelhantes quanto aos níveis socioeconômico e cultural. Uma contribuição importante é que dados dessa na- tureza, e de outras que dela decorrerem, possam melhorar a compreensão das relações entre as variáveis e oferecer elementos úteis ao planejamento de medidas visando à manutenção, melhora e promoção do bem-estar físico e psicológico das mulheres acometidas de câncer de mama.

Dessa maneira, pode-se realçar que pesquisas visando a analisar o impacto de uma doença na qualidade de vida de um grupo são de grande importância à sociedade, pois ajudam a direcionar a atenção da saúde pública para a melhora de seus serviços em benefício da população.

Merece destaque a importante atuação dos Grupos de Apoio na sociedade que assistem às mulheres acometidas pelo câncer de mama, pois além de proporcionarem informações sobre a doença, prevenção e suporte, trazem esperança para o enfrentamento do problema. O impacto positivo desses Grupos é o fato de serem compostos por mulheres que, na sua maioria, passaram pelo mesmo problema, tornando sua atuação altamente fidedigna.

\section{Colaboradores}

SFAP Simeão trabalhou no delineamento, análise e interpretação dos dados e redação final; ICR Landro trabalhou na concepção, coleta de dados e redação final; MHS De Conti trabalhou na interpretação dos dados, metodologia e revisão crítica; MAN Gatti trabalhou na redação final e revisão crítica; WD Delgallo trabalhou na metodologia e redação final; A Vitta trabalhou na análise e interpretação dos dados, redação final e revisão crítica.

\section{Referências}

1. Jemal A, Siegel R, Ward E, Hao Y, Xu J, Murray T, Thun MJ. Cancer statistics, 2008. CA Cancer J Clin 2008; 58(2):71-96.

2. Brasil. Ministério da Saúde (MS). Instituto Nacional do Câncer. Estimativa de câncer: incidência de câncer no Brasil. [Internet]. 2008 [acessado 2009 fev 06]. Disponível em: http://www.inca.gov.br/estimativa/ 2008/index. asp?link=conteudo_view.asp\&ID=5.

3. Yurek D, Farrar W, Andersen BL. Breast cancer surgery: comparing surgical groups and determining individual differences in postoperative sexuality body change stress. J Consult Clin Psychol 2000; 68(4):697-709.

4. Broeckel JA, Thors CL, Jacobsen PB, Small M, Cox CE. Sexual functioning in long-term breast cancer survivors treated with adjuvant chemotherapy. Breast Cancer Res Treat 2002; 75(3):241-248.

5. Engel J, Kerr J, Schlesinger-Raab A, Sauer H, Holzel D. Quality of life following breast-conserving therapy or mastectomy: results of a 5 -year prospective study. Breast J 2004; 10(3):223-231.

6. Spagnola S, Zabora J, Brintzenhofeszoc K, Hooker C, Cohen G, Baker F. The satisfaction with life domains scale for breast cancer (SLDS-BC). Breast $J$ 2003; 9(6):463-471.

7. Sheppard LA, Ely S. Breast cancer and sexuality. Breast J 2008; 14(2):176-181.

8. Beckjord E, Campas BE. Sexual quality of life in women with newly diagnosed breast cancer. J Psychosoc Oncol 2007; 25(2):19-36. 
9. Ganz PA, Kwan L, Stanton AL, Krupnick JL, Rowland JH, Meyerowitz BE, Bower JE, Belin TR. Quality of life at the end of primary treatment of breast cancer: first results from the moving beyond cancer randomized trial. J Natl Cancer Inst 2004; 96(5):376-387.

10. Huguet PR, Morais SS, Osis MJD, Pinto-Neto AM, Gurgel MSC. Qualidade de vida e sexualidade de mulheres tratadas de câncer de mama. Rev Bras Ginecol Obstet 2009; 31(2):61-67.

11. Gasparelo C, Sales CA, Marcon SS, Salci MA. Percepções de mulheres sobre a repercussão da mastectomia radical em sua vida pessoal e conjugal. Cienc Cuid Saude 2010; 9(3):535-542.

12. Vianna AMSA. Breast reconstruction patients psychological assessment: a pilot study. Estud psicol 2004; $21(3): 203-210$.

13. Sanitt JS. Breast reconstruction: a patient's story. The Breast 2006; 15(Supl. 2):S31-S33.

14. Hill O, White K. Exploring women's experiences of TRAM flap breast reconstruction after mastectomy for breast cancer. Oncol Nurs Forum 2008; 35(1):8188.

15. Santos DB, Vieira EMV. Imagem Corporal de mulheres com câncer de mama: uma revisão sistemática da literatura. Cien Saude Colet 2011; 16(5):25112522.

16. Elder EE, Brandberg Y, Björklund T, Rylander R, Lagergren J, Jurell G, Wickman M, Sandelin K. Quality of life and patient satisfaction in breast cancer patients after immediate breast reconstruction: a prospective study. The Breast 2005; 14:201-208.

17. Conde DM, Pinto-Neto AM, Cabello C, Santos-Sa D, Costa-Paiva L, Martinez EZ. Quality of life in Brazilian breast cancer survivors age 45-65 years: associated factors. Breast J 2005; 11(6):425-432.

18. Bech P. Quality of life measurements in the medical setting. Eur Psychiatry 1995; 10(3):83-85.

19. Bullinger M, Anderson R, Cella D, Aaronson N. Developing and evaluating cross-cultural instruments from minimum requirements to optimal models. Qual Life Res 1993; 2(6):451-459.

20. Rabin EG, Heldt E, Hirakata VN, Fleck MP. Quality of life predictors in breast cancer women. Eur $J$ Oncol Nurs 2008; 12(1):53-57.

21. Lahoz MA, Nyssen SM, Correia GN, Garcia APU, Driusso P. Capacidade Funcional e Qualidade de Vida em Mulheres Pós-Mastectomizadas. Rev Bras Cancerol 2010; 56(4):423-430.

22. Makluf ASD, Dias RC, Barra AA. Avaliação da qualidade de vida em mulheres com câncer da mama. Rev Bras Cancerol 2006; 52(1):49-58.

23. Sales CACC, Paiva L, Scandiuzzi D, Anjos AC. Qualidade de vida de mulheres tratadas de câncer de mama: funcionamento social. Rev Bras Cancerol 2001; 47(3):263-272.

24. Pinheiro CPO, Silva RM, Mamede MV, Fernandes AFC. Participação em grupo de apoio: experiência de mulheres com câncer de mama. Rev Latinoam Enferm 2008; 16(4):733-738.

25. Gomes FA, Panobianco MS, Ferreira CB, Kebbe LM, Meirelles MCC. Utilização de grupos na reabilitação de mulheres com câncer de mama. Rev enferm UERJ 2003; 11(3):292-295.

26. Barbosa RCM, Ximenes LB, Pinheiro AKB. Mulher mastectomizada: desempenho de papéis e redes sociais de apoio. Acta Paul Enf 2004; 17(1):18-24.
27. Regis MF, Simões MF. Diagnóstico de câncer de mama, sentimentos, comportamentos e expectativas de mulheres. Rev Eletr Enf [periódico na Internet]. 2005 [acessado 2009 nov 11]; 7(1):81-86. Disponível em: http://www.fen.ufg.br/revista/revista7_1/pdf/ORIGINAL_08.pdf.

28. Ware JR JE, Sherbourne CD. The MOS 36-itemshortform health survey (SF-36). I. Conceptual framework and item selection. Med Care 1992; 30(6):473-483.

29. Ciconelli RM, Ferraz MB, Santos W, Meinão I, Quaresma MR. Tradução para a língua portuguesa e validação do questionário genérico de avaliação de qualidade de vida SF-36 (Brasil SF-36). Rev Bras Reumatol 1999; 39(3):143-150.

30. Conselho Nacional de Saúde. Resolução no 196 de 10 de outubro de 1996. [Internet]. [acessado 2009 out 25]. Disponível em: http://www.conselho.saude.gov. br/resolucoes/1996/Reso196.doc.

31. Azevedo RF, Lopes RLM. Concepção de corpo em Merleau-Ponty e mulheres mastectomizadas. Rev Bras Enferm 2010; 63(6):1067-1070.

32. Veiga DF, Campos FSM, Ribeiro LM, Archangelo Jr I, Veiga Filho J, Juliano Y, Sabino Neto M, Ferreira LM. Mastectomy versus conservative surgical treatment: the impact on the quality of life of women with breast cancer. Rev Bras Saude Mater Infant 2010; 10(1):51-57.

33. Ueda S, Tamaki Y, Yano K, Okishiro N, Yanagisawa T, Imasato M, Shimazu K, Kim SJ, Miyoshi Y, Tanji Y, Taguchi T, Noguchi S. Cosmetic outcome and patient satisfaction after skin-sparing mastectomy for breast cancer with immediate reconstruction of the breast. Surgery 2008; 143(3):414-425.

34. King MT, Kenny P, Sheil A, Boyages HJ. Quality of life three months and one year after first treatment for early stage breast cancer: influence of treatment and patient characteristics. Qual Life Res 2000; 9(7):789-800.

35. Batiston AP, Santiago SM. Fisioterapia e complicações físico-funcionais após tratamento cirúrgico do câncer de mama. Fisioter Pesq 2005; 12(3):30-35.

36. Battaglini C, Bottaro M, Dennehy C, Barfoot D, Shields E, Kirk D, Hackney AC. The effects of resistance training on muscular strength and fatigue levels in breast cancer patients. Rev Bras Med Esporte 2006; 12(3):139-143.

37. Nissen MJ, Swenson KK, Ritz LJ, Farrell JB, Sladek ML, Lally RM. Quality of life after breast carcinoma surgery: a comparison of three surgical procedures. Cancer 2001; 91(7):1238-1246.

38. Amichetti M, Caffo O. Pain after quadrantectomy and radiotherapy for early-stage breast cancer: incidence, characteristic and influence on quality of life. Results from a retrospective study. Oncology 2003; 65(1):23-28.

39. Parker P, Youssef A, Walker S, Basen-Engquist K, Cohen L, Gritz ER, Wei QX, Robb GL. Short-Term and Long-Term Psychosocial Adjustment and Quality of Life in Women Undergoing Different Surgical Procedures for Breast Cancer. Ann Surg Oncol 2007; 14(11):3078-3089.

Artigo apresentado em 06/09/2011

Aprovado em 09/08/2011

Versão final apresentada em 09/11/2011 